Article

\title{
A Portable Live Cell Culture and Imaging System with Optional Umbilical Bioreactor Using a Modified Infant Incubator
}

\author{
Malcolm Brinn 1,*, Said F. Al-Sarawi ${ }^{2}$, Tien-Fu Lu ${ }^{3}$, Brian J. C. Freeman 4,5, Jaliya Kumaratilake ${ }^{1}$ \\ and Maciej Henneberg ${ }^{1}$ \\ 1 School of Medicine, Discipline of Anatomy and Pathology, University of Adelaide, Adelaide SA 5000, \\ Australia; Jaliya.Kumaratilake@adelaide.edu.au (J.K.); Maciej.Henneberg@adelaide.edu.au (M.H.) \\ 2 School of Electrical and Electronics Engineering, University of Adelaide, Adelaide SA 5000, Australia; \\ alsarawi@eleceng.adelaide.edu.au \\ 3 School of Mechanical Engineering, University of Adelaide, Adelaide SA 5000, Australia; \\ tien-fu.lu@adelaide.edu.au \\ 4 Discipline of Orthopaedics and Trauma, School of Medicine University of Adelaide, Adelaide SA 5000, \\ Australia; brian.freeman@adelaide.edu.au \\ 5 Department of Spinal Surgery, Royal Adelaide Hospital, North Terrace, Adelaide, Adelaide SA 5000, \\ Australia \\ * Corresponding Author: malcolm.brinn@adelaide.edu.au
}

\begin{abstract}
Here, we present a staged approach for an innovative repurposing of a portable infant humidicrib into a live cell growth, observation, and imaging system. Furthmore, humidicrib can support different variations of "umbilical" bioreactors, and can be used to conduct electrophysiology experiments and in situ immunohistochemistry. Modifications incorporate a closed loop carbon dioxide $\left(\mathrm{CO}_{2}\right)$ concentration control system with umbilical $\mathrm{CO}_{2}$ and heating support for tailored bioreactors. The repurposing cost is inexpensive and allows for the continued observation and imaging of cells. This prototype unit has been used to continuously observe and image live primary neurons for up to 21 days. This demonstrates the repurposed units' suitability for use in tissue culture based research, particularly where modifications to microscopes are required or where sensitive manipulation outside of a standard incubator is needed.
\end{abstract}

Keywords: cell culture; bioreactor; live cell imaging; tissue engineering

\section{Introduction}

Cell culture ovens and microscopes can typically be found in all cell culture laboratories. For many researchers, cells may only need observation at the time of passaging or during short term experiments and for this purpose quick transfer or stage top incubation systems may be employed. However, in some instances, it is necessary to observe and image cells over extended periods or to use tailored equipment. Commercial live cell imaging systems can be useful but for some, the cost of the equipment may be prohibitive for occasional use, or warranty conditions may limit options. Repurposing of assets is one potential way in which this can be resolved, by reducing expenditure as well as outbalancing the accrued cost of asset decommissioning (Loomba 2014; Opperman 2015).

Infant humidicribs are essential devices used to support neonates who would otherwise have difficulty regulating and maintaining their own homeostasis (Burgess 1978). Advances in the design of many of these devices have enabled them to regulate oxygen and humidity whilst isolating the infant in an aseptic environment. In a hospital setting, failure of this type of equipment can lead to catastrophic outcomes, therefore, these devices are often pragmatically decommissioned early in their working life cycle. However, being complex devices we believe they retain significant inherent value for alternate use. Here we present the novel repurposing of a decommissioned infant 
humidicrib into a tailored portable imaging culture system that can support different variations of "umbilical" bioreactors in a non-critical setting.

\section{Materials and Methods}

A decommissioned humidicrib as manufactured by Drager (Drager Air-Shields Isolette ${ }^{\circledR}$ C100) was obtained exgratia from a local government hospital. Device dimensions (mm) were $1340 \mathrm{H}$ x 560 $\mathrm{D} \times 1160 \mathrm{~W}$ comprising a plexiglass enclosure with a raised bed. The bottom section contained a control module, heating element, a port of oxygen supply, and an internal humidification system (Figure 1). This particular model also has an optional height adjustable strut under the trolley frame allowing user variation to suit standing or sitting.

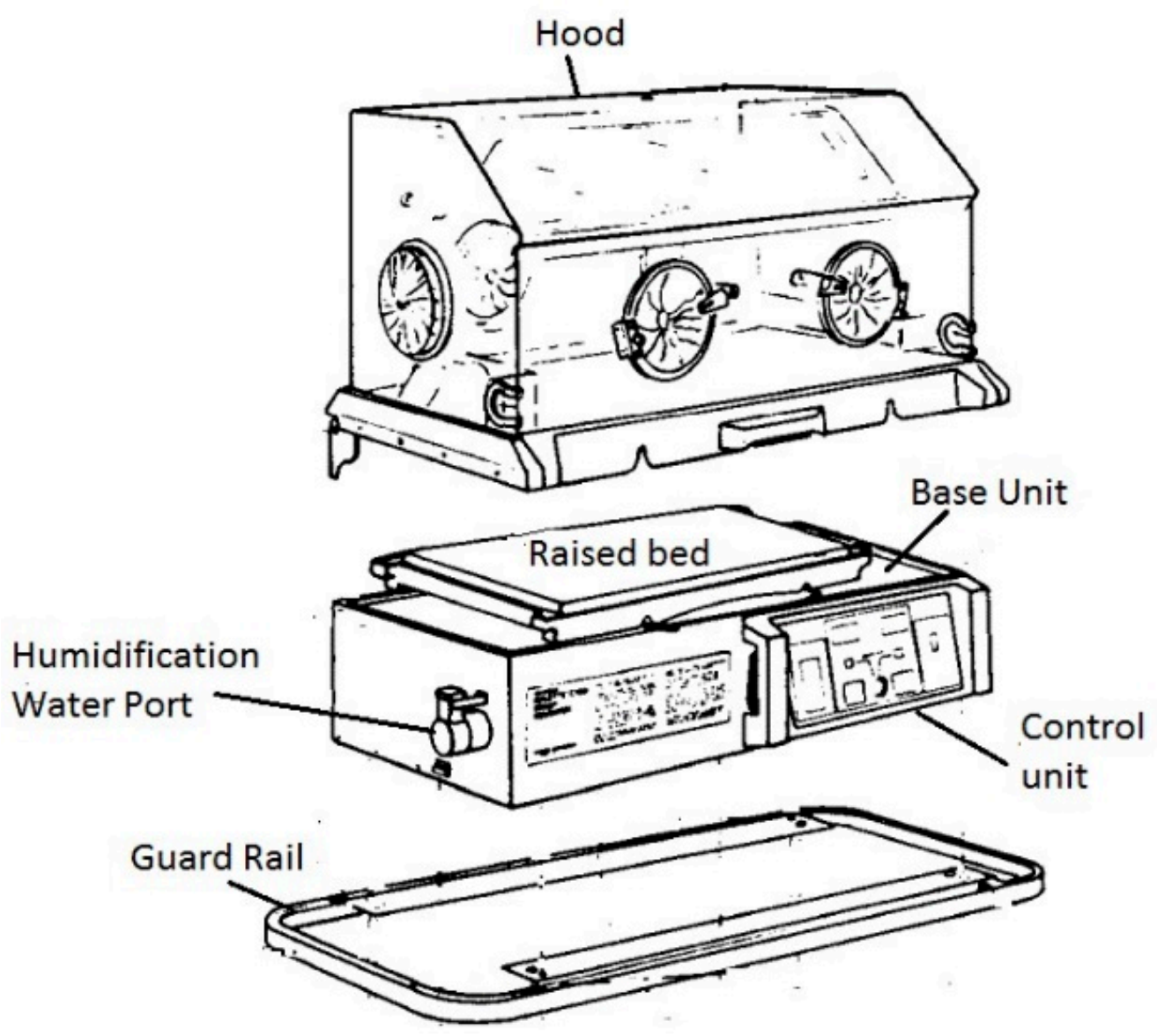

Figure 1. Adapted drawing (service manual) of the Drager Air-Shields Isolette ${ } . C 100$

Air is actively circulated across a heating element within the base and over an internal humidification tank and then passes into the interior. The plexiglass interior (shroud) of the infant incubator is isolated from the surrounding air by rubber seals. The control panel, has built-in skin or air temperature control devices, with digital displays (range 0 to $38.5^{\circ} \mathrm{C} \pm 0.5^{\circ} \mathrm{C}$ ) and visual and audible alarms. Access to the interior of the humidicrib is provided by air tight doors or iris entry ports. The raised infant bed can also be accessed by lowering the complete front panel. Gas can be introduced into the humidicrib through an external port (rear of unit - not shown) to which a gas bottle can be connected.

\subsection{Modifications to humidicrib}

An Olympus IMT2 inverted research microscope was mounted centrally between the front access ports of the humidicrib shroud, so that coaxial stage controls, revolving objective turret, field brightness, coarse and fine image adjustment controls, as well as a power switch could be accessed 
either by the right or left hand. Detachable access panels were cut into the centre rear and at the top/front of the humidicribs plexiglass shroud to allow the binocular shaft of the microscope to project neatly through the angled top of the plexiglass. A 3-Dimensional (3D) printer was used to construct tailored trim for the binocular shaft and silicone rubber matting was used to maintain internal seals.

Although the humidicrib had independent heating and humidity systems, it did not have an independent $\mathrm{CO}_{2}$ monitoring and control system. This was developed using a suitable regulator; gas approved 2W025-08 electric solenoid and an in-house established Arduino controller. When stationary, the gas supply could be either co-located onto the infant incubator making it portable or kept as a larger supply next to the unit. $\mathrm{CO}_{2}$ sensing was achieved through a fast acting $(20 \mathrm{~Hz})$ real time $\mathrm{CO}_{2}$ sensor (SprintIR GC-0017 0-20\%).



Figure 2 Diagram representing the $\mathrm{CO}_{2}$ gas connection direct to the incubator

$\mathrm{CO}_{2}$ gas enters the port and passes over an internal humidification and heating system. A fan circulates the gas up into the shroud where the SprintIR picks up the internal concentration. The closed Arduino circuit displays the $\mathrm{CO}_{2}$ concentration and activates the solenoid (default off).

Heating: The inner shroud temperature and humidity is maintained by the humidicribs existing infrastructure.

The $\mathrm{CO}_{2}$ controller comprised a closed loop relay circuit that was routed through a standard Arduino UNO circuit board controlling both the $\mathrm{CO}_{2}$ display powered via a standard printer USB cable and solenoid powered through a 12-volt adaptor (Figure 3). Arduino technology is an open source electronics platform intended for developing inexpensive interactive systems. Therefore, access to basic forms of Arduino circuit diagrams and source code was freely available from the internet under open access arrangements. 


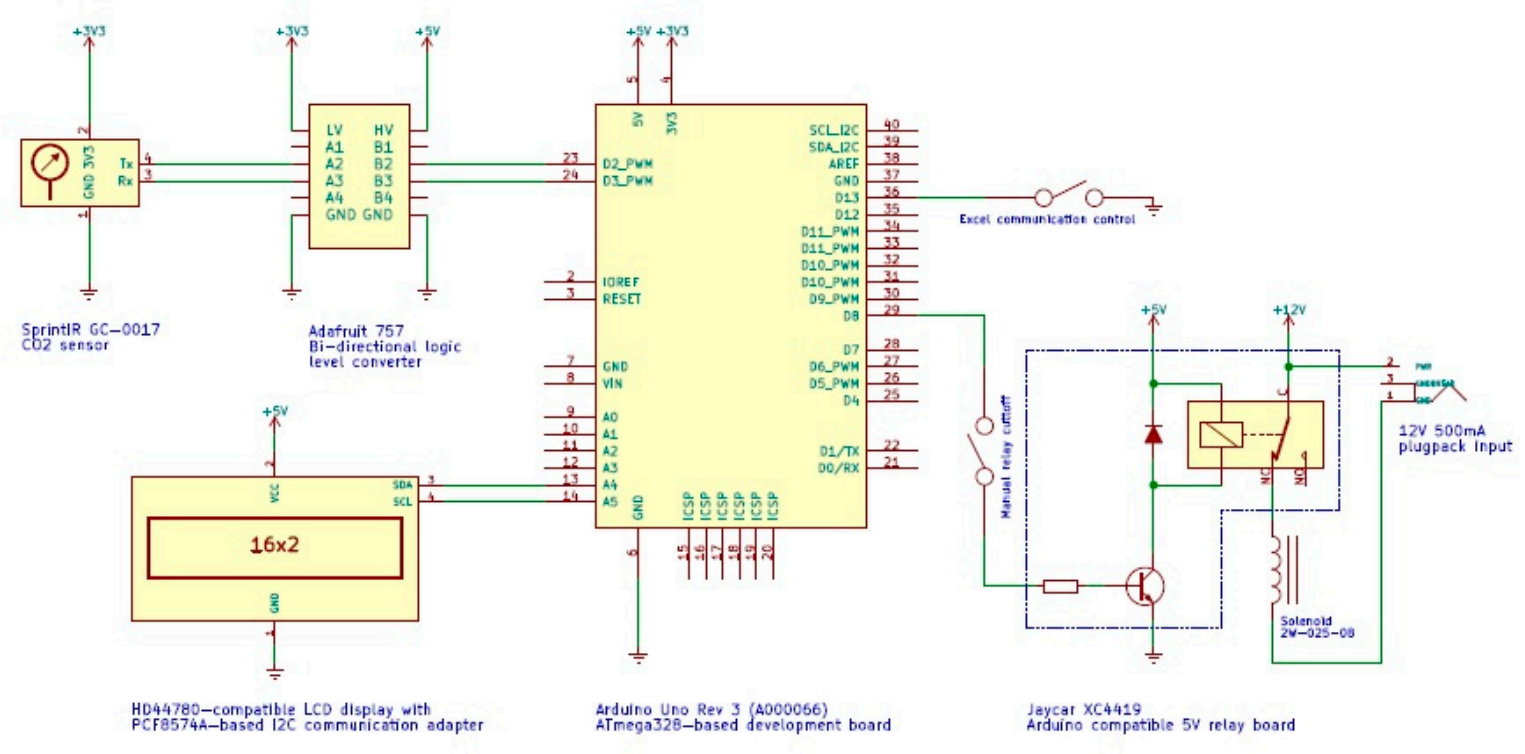

Figure 3. Arduino based circuit diagram for $\mathrm{CO}_{2}$ control

$\mathrm{CO}_{2}$ concentration is determined and controlled via a closed loop system. Air concentration is determined using a SprintIR 0-20\% $\mathrm{CO}_{2}$ sensor routed through a bi-directional logic level converter. Gas output is controlled through a $2 \mathrm{~W}-025-08$ gas solenoid (Default closed). The Arduino board controls the 5V relay, switching the solenoid on and off. A Liquid Crystal Display (LCD) shows \% $\mathrm{CO}_{2}$ levels. Data logging in CVS format is facilitated through an Excel spreadsheet which can be used to monitor flow over extended periods.

Adapted from www.pellinglab.com. Schematic modifications (incorporating the logic converter, independent $5 \mathrm{~V}$ relay board, $\mathrm{LCD}$ ) and final fabrication.

\subsection{Bioreactor development - Umbilical support (heating and gas)}

Pilot investigations highlighted that removal of cultures from the humidicrib for periods more than a few minutes caused a rapid loss of temperature and in the absence of $\mathrm{CO}_{2}, \mathrm{pH}$ variation. This was problematic for the use of equipment that could not be incorporated into the shroud and where cell/tissue homoeostasis was necessary. Provisioning the infant incubator to provide umbilical support to bioreactors rather than acting primarily as a portable culture oven, thus extends versatility and operational flexibility. The length of the umbilicus can be varied to suit requirements (Figure 4). 


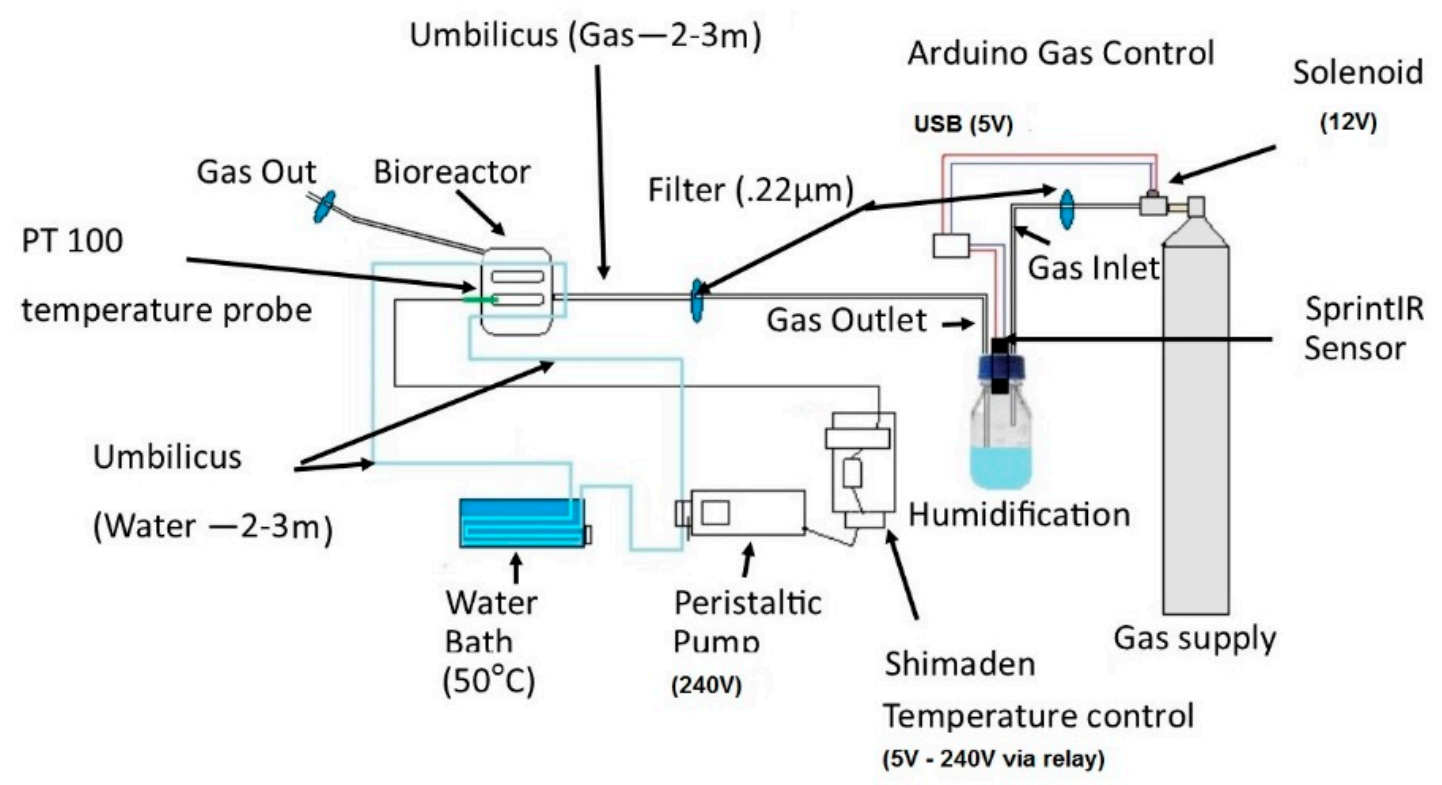

Figure 4: Diagram of heating and gas infrastructure for bioreactor use.

Heating: The PT100 temperature probe picks up the temperature and passes it to the Shimaden controller. At set points, the controller triggers a relay to open power to the peristaltic pump which circulates pre-heated water through the bioreactor.

Gas: The same Arduino control is used. However, gas from the solenoid is passed through a filter into a humidification container. Then the gas is filtered before entering the bioreactor. The operation of the controller remains the same.

Umbilical heating was provided through a water filled silicone tube $(6 \mathrm{~mm})$ which was weaved longitudinally through channels provided in each bioreactor and passed through a small sealed bath $\left(50^{\circ} \mathrm{C}\right)$. A PT100 temperature control probe in contact with the media provided feedback on temperature to a Shimaden Controller $\left( \pm 0.1^{\circ} \mathrm{C}\right)$, Schematic details are given Figure 5 .

Carbon dioxide was provision to the bioreactor was achieved by fitting $6 \mathrm{~mm}$ inlet and silicone outlet tubes and the existing $\mathrm{CO}_{2}$ SprintIR sensor into the lid of a standard glass $500 \mathrm{~mL}$ laboratory bottle. Before use, the assembly (excluding the sensor) was steam sterilized and then partially filled with sterile water under a laminar flow hood. Filtered $(0.22 \mu \mathrm{m}) \mathrm{CO}_{2}$ gas was then routed from the gas supply solenoid into the inlet tube. The outlet tube was tailored to appropriate length into the bioreactor $\mathrm{CO}_{2}$ port. A filter was used on the outlet tube, but care was taken to place this close to the bioreactor as humidity over time could cause the filter to block. 


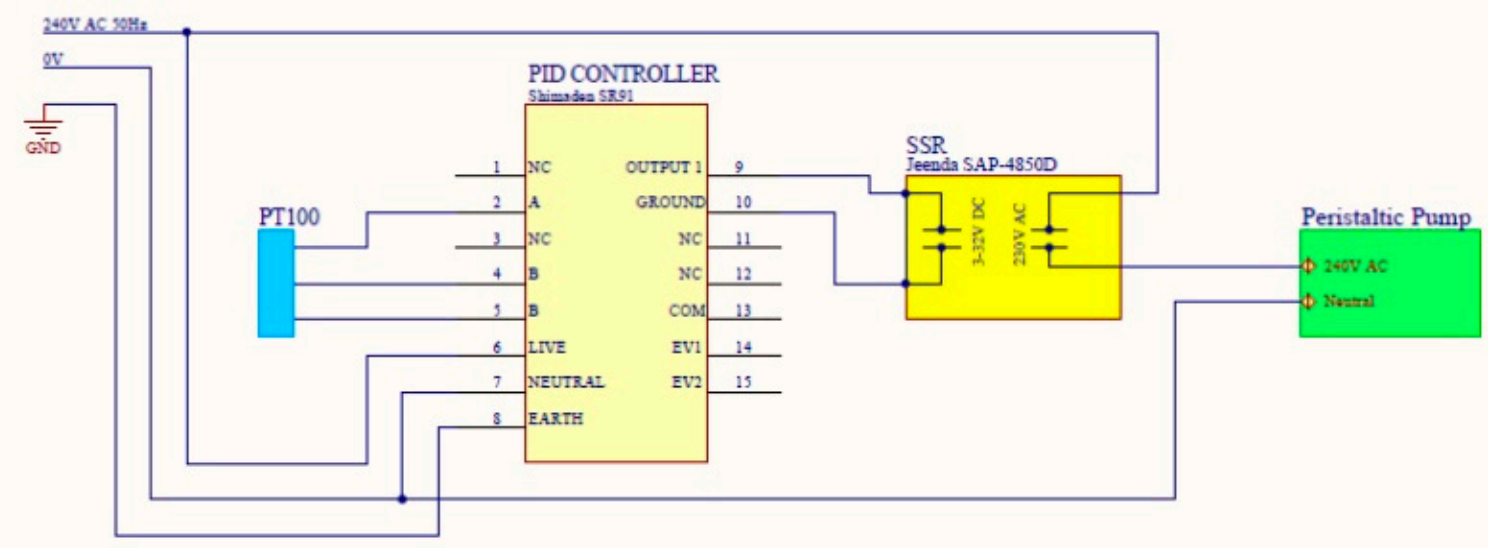

Figure 5: Schematic wiring diagram of the closed loop heating system.

Culture medium temperature is determined using a PT100 temperature sensor that is wired to a Shimaden SR91 proportional integral derivative (PID) controller. The PID triggers the solid state relay (SSR) to provide on/off power to a peristaltic pump that circulates the heated water. The Shimaden controller can be programmed with user-defined minimum and maximum set points and has built-in fuzzy logic. Therefore, over time the controller determines the optimum turn on/off ranges to mitigate overshoot of temperature.

\subsection{Bioreactor Design, Manufacture, and Assembly}

Here three bioreactors are presented (Figures 6 and 7), which were developed in series for the following experiments. The first example, a basic 3D printed version with the base plate was developed for culturing, monitoring and imaging of motor neurons. The second example, a modification separated the bioreactor component into two parts, with the lower section being machined and used with multiple different top sections. This modification achieved standardisation of the umbilical heating system, improved in situ immunostaining capability and facilitated material choice flexibility of the top section for different experiments such as stretching of axons.

All bioreactors were designed using Autodesk ${ }^{\circledR}$ Inventor $^{\circledR}$ Professional software 2017 and were for use with the inverted microscope. All devices were manufactured internally at the University Engineering Prototyping Laboratory. Each bioreactor differed in material selection and method of manufacture. However, all bioreactors used the same supporting infrastructure for both gas and heating. Length and width (mm) were standardized to $130(\mathrm{~L}) \times 100(\mathrm{~W})$. Height varied according to requirements. All designs of the bioreactor shared the key basic requirements of being non-toxic, sterilizable, and leak proof. Each featured two tissue culture medium/cell containment wells that accommodated removable standard $60 \times 24 \mathrm{~mm}$ cover glasses as viewing bases, external culture medium heating channels and provision to use a temperature probe in direct contact with the medium (Figure 6B). A standardised base plate for all versions of the bioreactor was machined from a $3 \mathrm{~mm}$ thick stainless steel sheet (Figure 6C). 
A

C

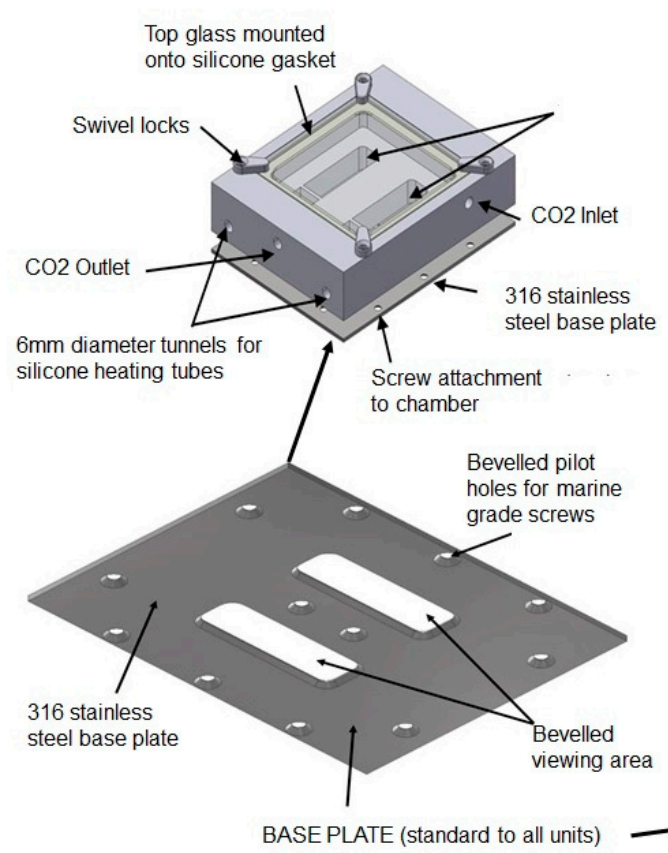

B

$6 \mathrm{~mm}$ diameter tunnels for silicone heating tubes

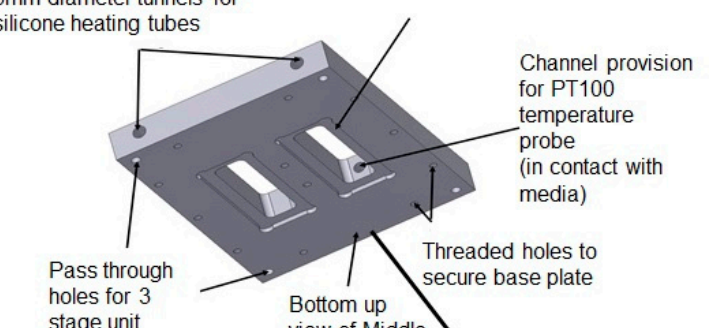
view of Mid Section

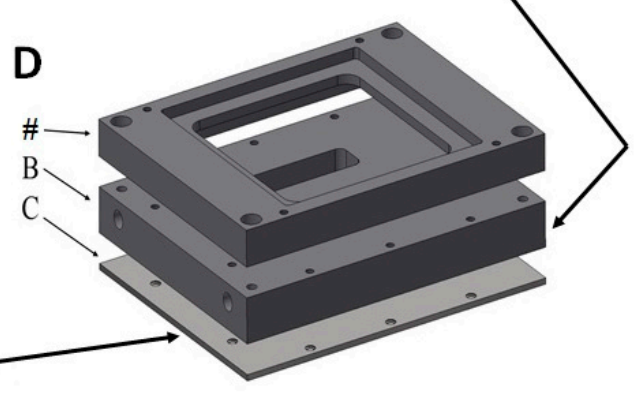

Figure 6: Basic design of bioreactors. A Top: Standard 1- piece 3D filament (Acrylonitrile Butadiene Styrene ABS) printed bioreactor with 316 stainless steel base plate B: Middle section of a three-piece bioreactor (including the 316-stainless steel base plate) machined from Polytetrafluoroethylene (PTFE). The top section on these units could be varied. C: The standard base plate common to all units D: Highlighting the 3-piece bioreactor assembly. \#: The Top and Middle sections of the 3-piece bioreactor are similar to the one-piece design when assembled. However, the middle section was machined from PTFE and the top section 3D filament printed with ABS.



Figure 7: Sagittal view example of a more advanced bioreactor (top aluminium machined (PTFE coated) bottom section machined from solid PTFE). Standard base plate not shown. 
This bioreactor incorporates many specialised features. The top section could be removed to conduct electrophysiology experiments and in situ immunohistochemistry. It also incorporates axon stretch technology adapted from (Loverde et al. 2011a). The lower (also shown 6B) incorporates a pilot version of an autofeeding system designed to prevent drying of the culture wells. The heating channels are running through the lower chamber on either side and between the medium containing wells (not visible in this view). Cover glasses can be removed for immunohistochemistry processing.

\subsection{Basic Live Cell Imaging}

Images obtained from this system are dependent both on the quality of the microscope and objectives, as well as on the imaging method. The Olympus IMT2 microscope used for our experiments was ideally suited for this task. We had both the option to use a commercial camera attached to the front port of the microscope or to use an ocular camera (Dino eyepiece camera AnMo Electronics Corporation). Attaching a camera to the front port of the microscope required the correct Olympus fitting for the camera but no calibration was readily achievable. However, the Dino eyepiece could be inserted direct into one of the two ocular viewing ports and taped into orientation. The image could then be projected to screen and captured via the DinoCapture sofware. Captured images can be calibrated and enhanced through the sofware (Figure 8B).

\section{Results}

\subsection{Humidicrib conversion results}

The humidicrib's internal heating system was sufficient to maintain cells in standard culture flasks. The internal shroud temperature of the infant incubator remained stable within specification. However, as with fixed culture incubators, it was essential to ensure the temperature of solutions was pre-set to operational requirements before placing cells into the hood. The large space within the humidicrib together with the mounting of an inverted research microscope and a digital camera enhanced functionality. The cutting of the front of the plexiglass shroud and fixing of custom made trim made it possible for the operation of the microscope via the front ports of the shroud without disruption to the cells.

Modifications to incorporate $\mathrm{CO}_{2}$ control direct into the shroud provided stand-alone capability. A reasonably large gas cylinder could be mounted onto the trolley making the unit fully portable. Alternatively, the unit could be plugged into a gas source or to a larger cylinder at the location when in use. The SprintIR sensor operated within specifications, and the Arduino controller triggered the solenoid to open (default closed) as required to maintain $\mathrm{CO}_{2}$ levels at the programmed concentration (in our case 5\%). The gas supply entered the bottom section of the incubator through a built in filter and humidification system; there was a slight delay in sensor receipt of the gas concentration. Following door opening, this does cause the unit to briefly overshoot gas concentration by as much as $2 \%$ for a $10-30$ second period.

The above modifications converted the decommissioned humidicrib into a portable culture oven capable of conducting continuous observation and imaging for research, particularly those that require continuous monitoring and sensitive manipulations.

\subsection{Bioreactor development- results}

Direct supply of $\mathrm{CO}_{2}$ to the bioreactor (via a humidification tank) reduced the consumption of $\mathrm{CO}_{2}$. Some problems occurred with the humidification system blocking filters, which were resolved through extending the $\mathrm{CO}_{2}$ line length and by placement of the post humidification filter closer to the bioreactor.

The internal heating system of the humidicrib was sufficient to maintain internal plexiglass shroud temperature but it a longer time to bring the inner temperature of the bioreactor to $37^{\circ} \mathrm{C}$. Supplemental heating of the bioreactor worked exceptionally well and maintained the temperature of the culture medium at $37^{\circ} \mathrm{C} \pm 0.1^{\circ} \mathrm{C}$. When both heating systems were in operation, the temperature of the culture medium remained very stable. 


\subsection{Repurposing Costs}

The total cost of repurposing the humidicrib is hard to estimate beyond the cost of supplies and materials (Table 1). The cost of labour will depend on specific skills of the staff involved and their commitment to the project. The cost of bioreactors will vary considerably based on production methods. 3D printing bioreactors once designed can be typically produced for under AU\$50.00. Costs for imaging will vary according to method used. A Dino eyepiece camera can be purchased for AU\$390.00.

Table 1: Equipment requirements for $\mathrm{CO}_{2} /$ Air sensor and control automation

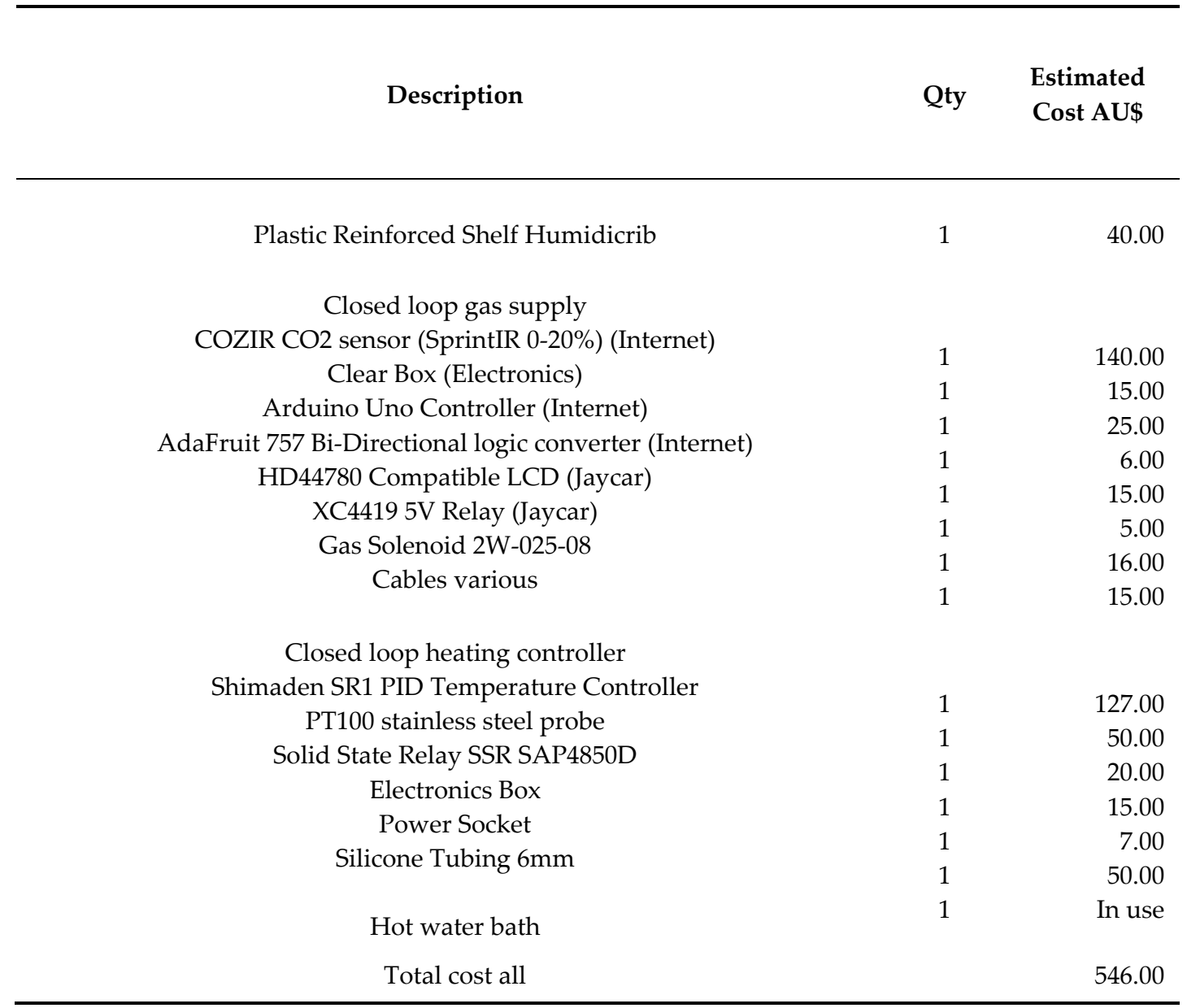

\subsection{Culture Use/Experience}

To date, fifty-four neuron cell cultures have been incubated in these bioreactors with experiment duration varying from 3 days to 21 days. The overall picture of the working unit is provided (Figure 8). Recent publication of some images obtained from live cells have been published elsewhere (Brinn et al. 2016) 


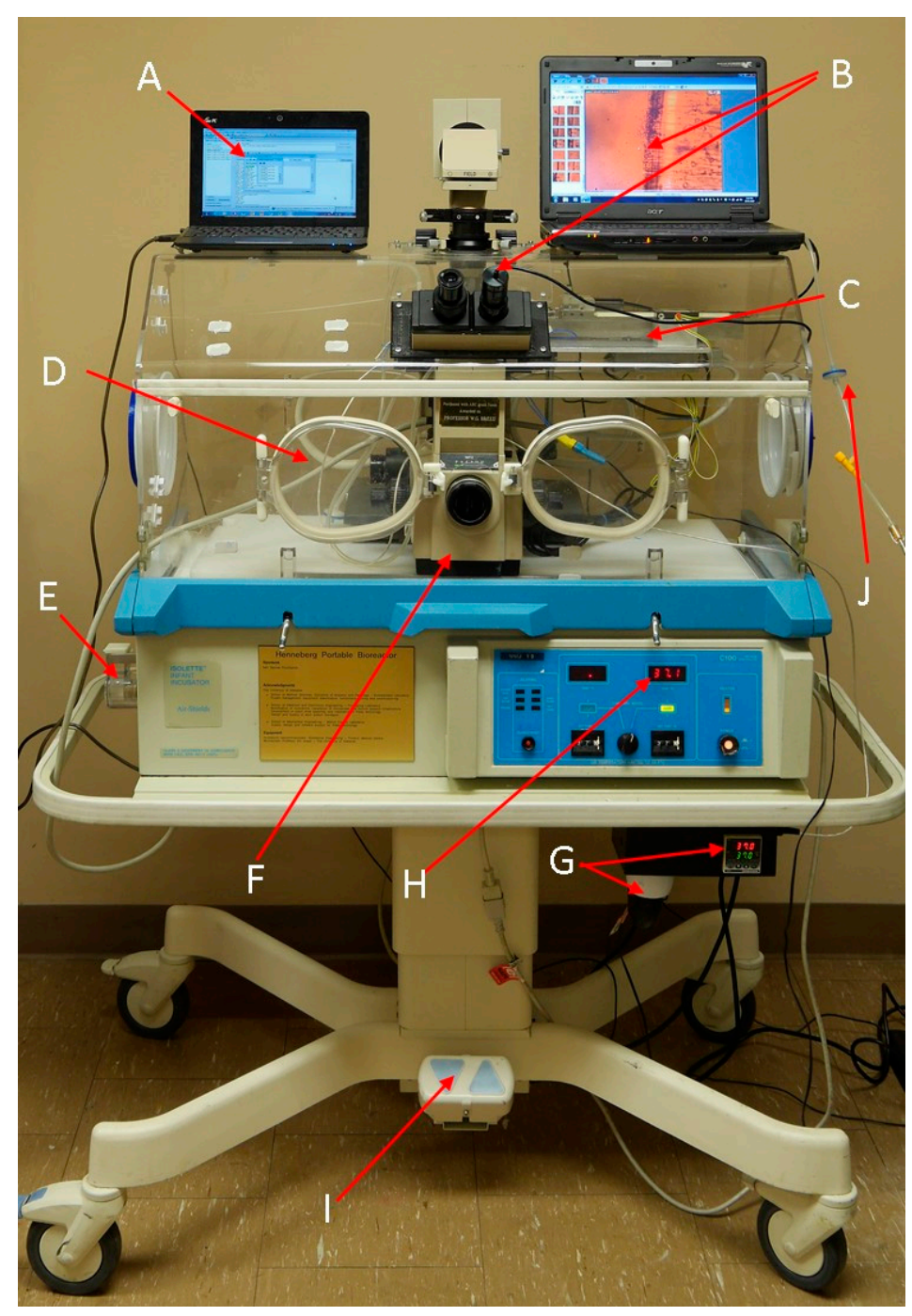

Figure 8. The complete system with assembled and functioning bioreactor in its controlled and protected housing (infant incubator)

The paediatric humidicrib provides a secondary barrier to minimise contamination, vibrations, and external movements during operation. The bioreactor was mounted onto the sample stage (C) of an Olympus IMT2 inverted microscope (F) with 4x, 10x, 20x and 40x objectives. Pre-warmed humidified $5 \% \mathrm{CO}_{2}$ filtered air $(0.22 \mu \mathrm{m})$ was delivered through the small humidification system and piped through sterile tubing directly to the bioreactor gas inlet port (J). Temperature control was maintained using a combination of the humidicrib temperature control system (to prevent fogging) (H), and an internal PT100 sensor attached to an external proportional-integral-derivative (PID) controller (l) activating power to the peristaltic pump to circulate heated water (not shown). Time lapsed imaging was captured using a Microscope Dino-Eye (USB) camera AM7023 connected to Dino-Lite v2 software (B). Laptop system monitoring software (A). A total of 6 intervention ports could be accessed to make adjustments (D).

\section{Discussion}

Live cell observation and imaging systems suitable for continuous use over several weeks are commercially available but expensive, and due to warranty issues modification can be problematic. Here, we have resolved this problem by repurposing an infant humidicrib and by developing an independent multipurpose bioreactor.

The humidicrib only required minor modifications to allow a variety of microscopes to be used. Once a suitable binocular port and removable top panels were cut into the plexiglass shroud it was a 
relatively simple exercise to lift off the shroud and install a variety of different microscopes. The advantage of this approach was that it allowed us to use our specifically tailored microscope with a number of onboard instruments. A situation that would not have been possible using a commercial system.

There were also several advantages in using our own internal microscopes. Perhaps the most important was the enhanced functionality, but familiarity was also beneficial when working with instruments. Microscopes of course are delicate instruments and the risk of damage to the optics is heightened during movement. However, this must be balanced against the alternative options available. In our case, removing the shroud and electrically adjusting the height of the stand made this a simple process and limited the risk. Another key benefit was the humidicribs portability. Once the microscope was removed, it could be easily transferred to storage or to another laboratory allowing its reuse with another microscope.

The incorporation of an independent 3D printable bioreactor has been another useful feature. In our case, we now have several different versions for variation in experiments. However, we continue to experiment with designs and materials. Currently, we are printing in standard plastics overnight. However, PEEK is now available for use, and it would be our intention to use this in future bioreactor development as it is autoclavable.

The independent heating system presented in this paper works well with the water filled silicone tubing passing through a standard heated water bath via basic peristaltic pump. The controller uses a standard relay and these can be assembled into a black box and wired by an electrician in less than an hour. The gas controller is built using Arduino technology and a standard gas solenoid, both of which are robust. Although assembly of the Arduino controller is not difficult, most electronics stores can provide this assembly service for a small fee. Source code to run the gas controller was originally written by Andrew Pelling and is available for download (www.pellinglab.net.au). We have subsequently added to the functionality and enclosed the full source code to allow operation of the device.

Equipment failure is of course always an issue. However, the dependency on the humidicrib is limited and heating elements within the unit can always be replaced with alternatives by a qualified electrician.

Although the unit can work as a standard incubator using standard cultureware, it does not have the insulation or advanced decontamination features available in purpose built units. However, the repurposing cost is inexpensive, and these units are frequently decommissioned.

Lastly, prior to use, it is recommended that appropriate electrical safety checks are made to the humidicrib. $\mathrm{A} \mathrm{CO}_{2}$ warning sensor should also be present where culture ovens or this device is in use.

\section{Conclusion}

Infant incubators have inbuilt features that can be adapted for the growth of tissue cultures. Therefore, they could be modified into live cell imaging systems. Also, this apparatus can accommodate advanced technology. The cost in adopting these modifications may be inexpensive in comparison to new equipment purchase.

Acknowledgement: We gratefully acknowledge the donation of the decommissioned infant incubator provided by Flinders Medical Centre, South Australia, the technical assistance of Mr Chris Leigh, School of Medicine, University of Adelaide and Mr Ian Linke, Mr Brandon Pullen, Mr Aubrey Slater, Mr Alban O'Brien, Mr Hayden Westell and Mr Danny Di Giacomo School of Electrical and Electronic Engineering, University of Adelaide. 


\section{References}

Brinn, MP, O'Neill, K, Musgrave, I, Freeman, BJC, Henneberg, M \& Kumaratilake, J 2016, 'An optimized method for obtaining adult rat spinal cord motor neurons to be used for tissue culture', J Neurosci Methods, vol. 273, pp. 128-137.

Burgess, M, (President), 1978, 'Abstracts of Papers Presented at The 11th Annual Meeting', Journal of Paediatrics and Child Health, vol. 14, no. 2, pp. 109-127.

Loomba, APS 2014, 'Repurposing medical devices for LRCs; Sustainability considerations', Engineering, Technology and Innovation (ICE), 2014 International ICE Conference on, 23-25 June 2014, pp. 1-6.

Loverde, JR, Ozoka, VC, Aquino, R, Lin, LS \& Pfister, BJ 2011a, 'Live imaging of axon stretch growth in embryonic and adult neurons', J Neurotrauma, vol. 28, no. 11, Nov, pp. 2389-2403.

Opperman, LA 2015, 'Anatomist executive talks device development', Biomedical Instrumentation and Technology, vol. 49, no. 2, pp. 125-127.

\section{Supplementary Material}

\section{CO2_PID_Controller_Arduino Source Code (Open access source code)}

// Arduino IDE version used for development/compatibility reasons: 1.0.6

// https://www.arduino.cc/en/Main/OldSoftwareReleases\#previous

//Acknowledgement to Andrew Pelling @ www.pellinglab.net for original version

//Modifications: Danny Di Giacomo and Malcolm Brinn - University of Adelaide www.adelaide.edu.au

\section{$/ *$}

Humidifier and $\mathrm{CO} 2$ gas control via $12 \mathrm{~V}$ normally closed solenoid and $\mathrm{CO} 2$ Sensor.

CO2 Meter (SprintIR 0-20\% GC-0017):

http://www.co2meter.com/collections/co2-sensors/products/sprintir-100-percent-co2-sensor

also need the latest COZIR library, download from this thread (Using Cozir 0-1-01.zip in this code):

http://forum.arduino.cc/index.php?topic $=91467.0$

CO2 supplied by BOC Gas

Arduino UNO Pin Connections:

Pin 2,3: Tx/Rx from CO2 //Pin 2 arduino goes to Tx on Sensor Pin 4

Pin \#5: 
Pin \#6:

Pin \#7:

Pin \#8: Solenoid Relay

Pin \#9:

SCL/SDA Pins: 12C Display

$* /$

\section{/* CO2 CONTROL /////////}

Control works by reading the sensor (CO2) 3 times and averaging (to flatten out noise).

The measurement is compared to a user-defined SETPOINT. If the CO2 is below the setpoint, the solenoid opens allowing the flow of $\mathrm{CO} 2$ into the incubator. Once the $\mathrm{CO} 2$ content comes within a user-defined THRESHOLD of the setpoint, a stepping cycle begins. The solenoid opens for a user defined DURATION and

then closes. The open/closed cycle repeats until the setpoint is reached.

The cycles allow the system to step up to the setpoints.

Setpoints, thresholds and durations MUST be set according to your own system by TRIAL AND ERROR. May need to increase/decrease depending on size/shape of incubator, $\mathrm{CO} 2$ flow rate, etc.

$* /$

float CO2Setpoint $=1.0 ; \quad / /$ Setpoint $\mathrm{CO} 2$ level in $\% 5$

float CO2Threshold $=0.85$; / Threshold to switch to stepping control in \%/100

int SolenoidOnTime $=5000$; // Duration time in milliseconds

// CO2 Sensors

\#include "SoftwareSerial.h"

\#include "cozir.h"

// Cozir library info/download: http://forum.arduino.cc/index.php?topic=91467.0

// Excel logging configuration

// http://www.robertovalgolio.com/sistemi-programmi/arduino-excel

\#include $<$ rExcel.h $>$

long idx $=0$; // index

int outputTiming $=1000 ; \quad / /$ packet sending timing in $\mathrm{ms}$, determines the output timing

rExcel myExcel; $\quad$ // class for Excel data exchanging

int save_loop_timer $=1$;

$/ *$

char worksheet[16]; // worksheet name

char range[16]; $\quad$ // range set

unsigned int row; // row

unsigned int column; // column

char value[16]; $\quad$ // written or read value

*/ 
// I2C LCD interface configuration

// http://forum.arduino.cc/index.php?topic=128635.0

// https://bitbucket.org/fmalpartida/new-liquidcrystal/downloads

// LiquidCrystal_V1.2.1.zip library used

\#include $<$ Wire.h $>$

\#include $<$ LCD.h $>$

\#include $<$ LiquidCrystal_I2C.h $>$

\#define I2C_ADDR 0x3F

\#define BACKLIGHT_PIN 3

\#define En_pin 2

\#define Rw_pin 1

\#define Rs_pin 0

\#define D4_pin 4

\#define D5_pin 5

\#define D6_pin 6

\#define D7_pin 7

LiquidCrystal_I2C lcd(I2C_ADDR,En_pin,Rw_pin,Rs_pin,D4_pin,D5_pin,D6_pin,D7_pin);

///////* RELAY *///////

int Solenoid = 8; $\quad$ // Relay for controlling $12 \mathrm{~V}$ solenoid valve

///////* SPRINTIR COZIR CO2 SENSOR *///////

SoftwareSerial nss(2, 3); // Rx, Tx - Pin 3,4 on sensor

COZIR czr(nss);

float SingleCO2, $\mathrm{CO} 2=0$;

float multiplier $=0.001 ; \quad / / 10 / 10000$ (Hardware multiplier/ppm conversion).

float reading $=0$;

void $\operatorname{setup}()$

\{

Serial.begin(9600); / Start serial port

czr.SetOperatingMode(CZR_POLLING); // Start the CO2 sensor and put into POLLING mode

pinMode(Solenoid, OUTPUT); / / Sets pin for controlling solenoid relay

digitalWrite(Solenoid, LOW); $\quad$ // Set LOW (solenoid closed off)

lcd.begin(16,2);

lcd.setBacklightPin(BACKLIGHT_PIN,POSITIVE);

lcd.clear();

lcd.setBacklight(HIGH);

lcd.home();

pinMode(13, INPUT_PULLUP);

// delay(500); // Wait

\} 
void loop ()

\{

// Read CO2 sensor 3 times and determine the average.

for (int $\mathrm{i}=0 ; \mathrm{i}<3 ; \mathrm{i}++)\{$

SingleCO2 $+=$ czr.CO2() * multiplier;

\}

$\mathrm{CO} 2=$ SingleCO2 $/ 3$;

SingleCO2 $=0$;

// Write $\mathrm{CO} 2 \%$ to LCD

lcd.setCursor $(0,0)$;

lcd.print("CO2 level: ");

lcd.setCursor $(10,1)$;

lcd.print $(\mathrm{CO} 2,2)$;

lcd.print(" \%");

// If switch on GPIO 13 low, enable Excel logging

if $($ digitalRead $(13)==$ LOW $)\{$

static unsigned long loopTime $=0$;

static unsigned long time $1=0$;

loopTime $=$ millis () ;

// Output Task

// Arduino acts as client making requests to Excel

// instructions performed each outputTiming ms

if $(($ loopTime - time 1$)>=$ outputTiming $)\{$

time1 = loopTime;

myExcel.write("Template", "A5", "\%time\%");

// write time to sheet "Template" cell A5

myExcel.write("Template", "B5", CO2, 2);

$/ /$ write $\mathrm{CO} 2 \%$

myExcel.writeIndexed("Template", idx+11, 1,"\%date\%"); // idx is zero initially, write date to row $\mathrm{idx}+11$, column 1

myExcel.writeIndexed("Template", idx+11, 2,"\%time\%");

myExcel.writeIndexed("Template", idx+11, 3, idx);

myExcel.writeIndexed("Template", idx+11, 4, CO2, 2);

$\mathrm{idx}++$;

// Save spreadsheet every 60 seconds

save_loop_timer++;

if (save_loop_timer $==60$ ) \{ 


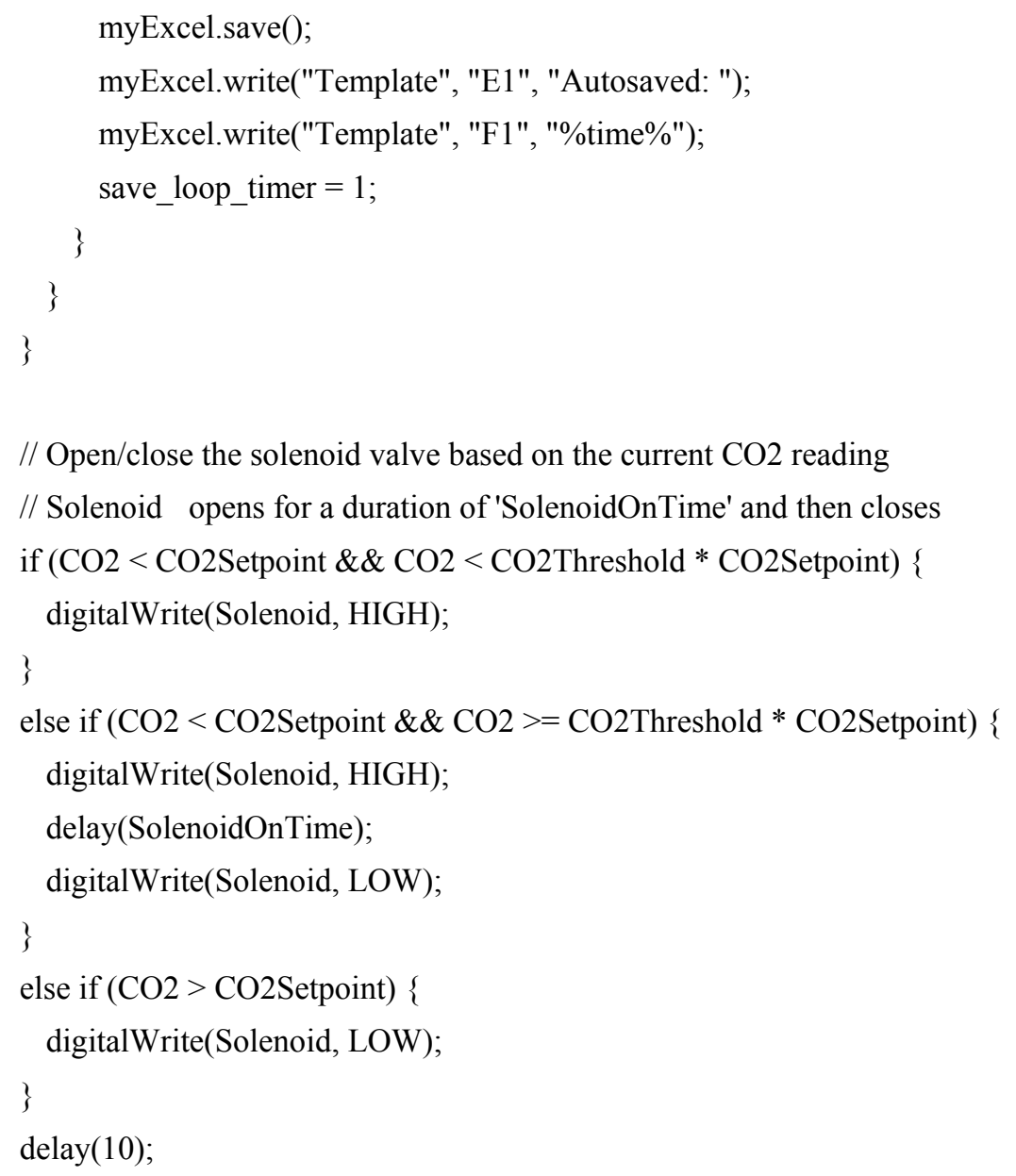

2017 by the authors; licensee Preprints, Basel, Switzerland. This article is an open access article distributed under the terms and conditions of the Creative Commons by Attribution (CC-BY) license (http://creativecommons.org/licenses/by/4.0/). 